\title{
SBC2009-206670
}

\section{DEVELOPMENT AND BIOCOMPATIBILITY CHARACTERIZATION OF A BIOMEMS SENSOR FOR MONITORING THE PROGRESSION OF FRACTURE HEALING}

\author{
Brandon G. Santoni (1), Rohat Melik (2), Emre Unal (2), Nihan Kosku Perkgoz (2), Debra A. \\ Kamstock (3), Stewart D. Ryan (3), William S. Dernell (4), Hilmi Volkan Demir (2), Christian M. \\ Puttlitz (1)
}

(1) Department of Mechanical Engineering, School of Biomedical Engineering, Colorado State University, Fort Collins, CO

(3) Department of Clinical Sciences, James L. Voss Veterinary Medical Center, Colorado State University, Fort Collins, CO

\author{
(2) Department of Electrical and Electronics \\ Engineering, Department of Physics, \\ Nanotechnology Research Center, Institute of \\ Materials Science and Nanotechnology, \\ Bilkent University, Ankara, Turkey
}

(4) Department of Veterinary Clinical Sciences, Washington State University, Pullman, WA

\section{INTRODUCTION}

Orthopaedic extremity injuries present a large medical and financial burden to the United States and world-wide communities [1]. Approximately six million long bone fractures are reported annually in the United States and approximately $10 \%$ of these fractures do not heal properly. Though the exact mechanism of impaired healing is poorly understood, many of these non-unions result when there is a communited condition that does not proceed through a stabilized healing pathway [2]. Currently, clinicians may monitor healing visually by radiographs, or via manual manipulation of the bone at the fracture [3]. Unfortunately, the course of aberrant fracture healing is not easily diagnosed in the early period when standard radiographic information of the fracture is not capable of discriminating the healing pathway. Manual assessment of fracture healing is also an inadequate diagnostic tool in the early stages of healing [4].

To overcome these shortcomings, we have developed a radio frequency (RF) strain sensor design that takes advantage of advances in micro-electro-mechanical systems (MEMS) technology. The inductively-powered sensor is intended to monitor the surface bending strains on implanted hardware. Our prior work has included analytical and numerical analyses including RF finite element simulations and prototype fabrication that demonstrate the feasibility of our proposed approach [5,6]. Currently, second generation prototypes are being fabricated that represent fine tuning of the sensor geometry to enhance the signal quality and sensitivity. Prior to applied studies numerous challenges must be overcome if MEMS devices are to be successfully implemented for implantable medical applications. To date, the safety and performance of the materials that constitute our bioMEMS sensor have not been investigated. Thus, the scope of the current study was to investigate the site-specific biocompatibility and wound-healing response elicited against this novel device in a small animal model.

\section{MATERIALS AND METHODS}

MEMS Materials \& Sensor Development

The bioMEMS sensor investigated here is designed and implemented in spiral architecture (Fig. 1). Its $\mathrm{Si}_{3} \mathrm{~N}_{4}$ dielectric film is deposited on a silicon substrate by PECVD (plasma enhanced chemical vapor deposition). Subsequently, by photolithography and metal deposition, gold is deposited on $\mathrm{Si}_{3} \mathrm{~N}_{4}$ to finalize sensor fabrication. Sensor design parameters are presented in Table 1 , where $L_{c}$ and $W_{c}$ are the total length and width of the device, $N$ is the number of turns, $w$ is the coil width, $s$ is spacing between the segments in the spiral coil, $t_{\text {film }}$ is the thickness of the dielectric layer, and $t$ is the thickness of the metal layer.

In operation, this bioMEMS sensor exhibits a characteristic resonance frequency. By applying external loads to the device in a controlled fashion, the resonance frequency shifts as a result of deformation of the area of the dielectric layer under the applied load, leading to a large change in the capacitance of the device. By measuring the shift in resonance frequency, information on the load carried by the implanted fracture fixation plate, and thus insights into the progression of fracture healing, can be obtained. Preliminary ex vivo data aside, we have yet to rigorously address the issue of biocompatibility of our bioMEMS device.

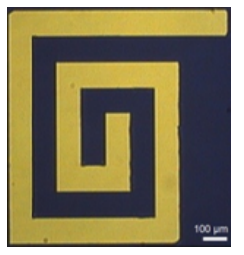

Fig. 1. Top-view micrograph of our fabricated bioMEMS sensor. 
Table 1.

bioMEMS Sensor Design Parameters.

\begin{tabular}{ccccccc}
\hline$L c(\mu \mathrm{m})$ & $W c(\mu \mathrm{m})$ & $N$ & $w(\mu \mathrm{m})$ & $s(\mu \mathrm{m})$ & $t_{\text {film }}(\mu \mathrm{m})$ & $t(\mu \mathrm{m})$ \\
\hline 900 & 900 & 2 & 100 & 100 & 0.1 & 0.1 \\
\hline
\end{tabular}

\section{Biocompatibility Experimental Design}

New Zealand White Rabbits were chosen as an appropriate animal model because of their size and ability to house multiple bioMEMS chips. A total of four rabbits each implanted with 4 MEMS devices and 2 control material implants (6 implants/rabbit; 16 MEMS and 8 controls total) were used to investigate the biocompatibility of the device in accordance with ASTM Standards F981-04 and F763-04. Animals were humanely euthanized six months post-operatively at which time critical gross pathology and microscopic evaluation of the implant sites for an implant-associated tissue reaction was pursued.

\section{Surgical Procedure}

Prior to surgery, implant materials were sterilized via two cycles of autoclave for $25 \mathrm{~min} / 10 \mathrm{~min}$ dry at $121^{\circ} \mathrm{C}$. The surgical sites were denuded of all hair on both sides of the spinal column. The skin was swabbed lightly with diluted alcohol and dried prior to sample implantation. Each rabbit received six $(n=6)$ sterilized samples (four test materials and two controls) each implanted in individual sites of the lumbar paravertebral musculature. Two bioMEMS chips $(5 \mathrm{~mm} x$ $5 \mathrm{~mm}$ ) and one control specimen (aluminum oxide, $\mathrm{Al}_{2} \mathrm{O}_{3}$; ASTM F603-00(2000)) were placed parallel to and on either side of the spinal column approximately 2.5 to 5 from midline and $2.5 \mathrm{~cm}$ apart from each other resulting in a total of 3 implants per side.

\section{Euthanasia and Implant Site Analysis}

Animals were euthanized six months postoperatively by intravenous injection of sodium pentobarbital. The tissue surrounding and overlying each implant, both for bioMEMS test material and the $\mathrm{Al}_{2} \mathrm{O}_{3}$ control material, was macroscopically evaluated for evidence of internal and external lesions in accordance with a semi-quantitative scoring system developed in our laboratory. Each implant was then removed with an intact envelope $(\sim 4 \mathrm{~mm})$ of surrounding tissue and fixed for 24 hours in $10 \%$ neutral buffered formalin. After fixation, each implant was removed from the tissue envelope and the tissue specimens were routinely processed, embedded in paraffin, sectioned at $5 \mu \mathrm{m}$, and stained with Hematoxylin and Eosin (H\&E) for semiquantitative evaluation of the cellular and tissue response to the bioMEMS and control materials. Microscopic evaluation was performed by a single board certified pathologist (D.A.K.) blinded to the treatment groups so as to avoid observer bias.

\section{RESULTS}

During convalescence, there were no complications resulting from the surgical procedure, no evidence of post-operative infection, and no mortality in the six-month survival period. At euthanasia, gross examination of tissue adjacent to the bioMEMS materials did not reveal any visible signs of adverse reactions manifested as external or internal lesions to the test materials. No infection or inflammation was grossly noted in the musculature surrounding implanted materials.

Microscopic examination of the H\&E stained slides confirmed that there was no adverse tissue reaction to the bioMEMS materials either immediately adjacent to or peripheral to the implantation site (Fig. 2). Examination of histological slides confirmed the absence of abnormal macrophage or lymphocytic cellular activity. Resultantly, inflammation scores for the bioMEMS and $\mathrm{Al}_{2} \mathrm{O}_{3}$ control materials were $0 \pm 0$ and $0 \pm 0$ (mean \pm stdev), respectively. Minimal fibrosis was noted surrounding both the control and bioMEMS materials $(1.0 \pm$ 0.5 and $0.94 \pm 0.24$, respectively) and the general toxicity score for the test and control materials was zero.
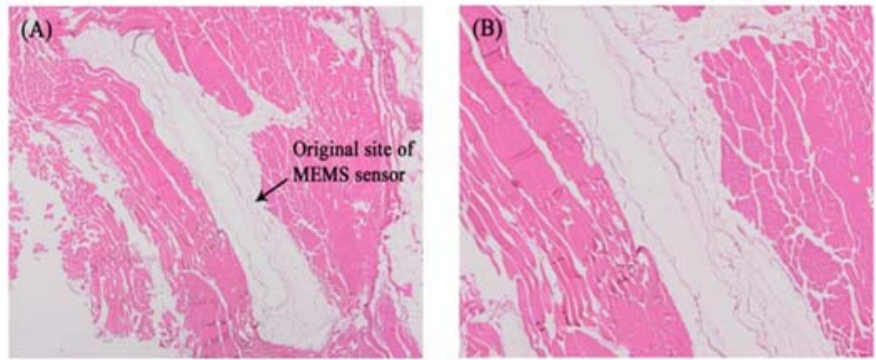

Fig 2. $2 x(A)$ and $4 x(B)$ images of the H\&E stained tissue adjacent to the implanted MEMS sensor. After six months, no evidence of inflammation or adverse tissue response was documented adjacent to any of the implanted MEMS materials.

\section{DISCUSSION}

Monitoring real-time fracture healing in vivo using MEMS technology is an attractive alternative to traditional radiographic and physical manipulation modalities as the implantable sensor output is quantitative in nature and far less subjective. However, prior to applied clinical studies, baseline biocompatibility of the implantable sensor must be thoroughly investigated and established. Using a rabbit model, Kotzar et al. [7] demonstrated that the common materials used in the construction of MEMS sensors, including silicon ( $\mathrm{Si}$ ), silicon dioxide $\left(\mathrm{SiO}_{2}\right)$, and silicon nitride $\left(\mathrm{Si}_{3} \mathrm{~N}_{4}\right)$, do not exhibit a quantifiable adverse tissue response after 12 weeks in vivo. Given that complex tibial fractures, for example, may take up to six months to heal completely, to monitor the complete course of osseous healing, implantable devices may be required to both function appropriately and demonstrate adequate biocompatibility for greater than 24 weeks in the body. Thus the present study sought to build on the previous work by focusing on the long-term biocompatibility of our bioMEMS device in sites relevant for a clinical application.

The preliminary results presented here demonstrate the sitespecific biocompatibility of the MEMS materials that we propose for use in monitoring bending strains on internal fixation devices that stabilize and promote fracture repair. Though these preliminary results are encouraging, additional studies are still required to definitively characterize the tissue responses that would be induced by our MEMS device in an osseous wound site as well as ensure continued sensor functionality in vivo for time periods similar to those required for complex fracture healing.

\section{CONCLUSION}

The findings presented here support further development and characterization of a fracture healing monitoring system based on implantable MEMS devices.

\section{REFERENCES}

[1] Heckman \& Sarasohn-Kahn, Bulletin, 1997; [2] Gaston \& Simpson, JBJS Br., 2007; [3] Simpson et al., Injury, 2000; [4] Webb et al., Injury,1996; [5] Melik et al., J Micromech Microeng, 18, 115017 2008; [6] Melik et al., IEEE Transactions on Electron Devices 55, 3459-3466 2008; [7] Kotzar et al., Biomaterials, 2002. 